\title{
Influência da perceção de apoio social no bem-estar psicológico em adolescentes portugueses
}

\section{The influence of the perceived social support on psychological well- being among portuguese adolescents}

\author{
E. Ramião, H. Fernandes, S. Quesado
}

ARTIGO ORIGINAL | ORIGINAL TITLE

\begin{abstract}
RESUMO
Apesar da sistemática investigação em torno da perceção de apoio social, poucos são os estudos que procuraram compreender a sua influência ao nível do bem-estar psicológico na adolescência. O presente estudo visa identificar possíveis influências da perceção de apoio social sobre as várias dimensões do bem-estar psicológico. A amostra do estudo foi de 283 estudantes portugueses, do terceiro ciclo e ensino secundário, entre os 12 e os 17 anos de idade, sendo $52,7 \%$ do sexo feminino. Foram usadas duas escalas de autorrelato para avaliar o bem-estar psicológico e o apoio social percebido. Existiram influências significativas das dimensões da perceção de apoio social (amigos, família, professores e apoio geral) nas dimensões do bem-estar psicológico, sendo que na maioria dos casos níveis baixos de perceção de suporte associaram-se a níveis menores ao nível do bemestar psicológico comparativamente aos grupos com perceção de suporte mais elevada. A perceção de apoio de suporte social veicula relações com o bem-estar psicológico, deixando o pressuposto de que o apoio percebido pelos adolescentes constitui uma variável que pode limitar o desenvolvimento de capacidades inerentes à realização positiva destes. Neste prisma, incentiva-se a sistematização de pesquisas sobre a temática.
\end{abstract}

Palavras-chave: perceção de apoio social, bem-estar psicológico, adolescência

\begin{abstract}
Even though there has been systematic research into the perception of social support, few studies have sought to understand their influence on adolescents' psychological well-being. This study aims to identify possible consequences of social support perception in the various dimensions of psychological well-being. The study sample consisted of 283 Portuguese students, ranging from grammar to secondary education, between 12 and 17 years old, being $52.7 \%$ were female. Two self-report scales were used to assess psychological well-being and perceived social support. Results showed significant influences of social support dimensions of perception (friends, family, teachers, and general support) in the dimensions of psychological well-being. Still, low levels of perceptions of support were also associated with lower levels of psychological well-being compared to those with higher perception support. The individual's perception of social support is related to psychological well-being. These results suggest that adolescents support is a variable that can limit the development of capacities inherent to their positive achievement. Further research needs to be conducted to systematize research on the topic.
\end{abstract}

Keywords: perceived social support, psychological well-being, adolescence

Submitted: 12.05.2019 | Accepted: 10.24.2020

Eduarda Z. Ramião. Psicóloga, Laboratório Neuropsicofisiologia, Faculdade de Psicologia e Ciências de Educação da Universidade do Porto. Porto, Portugal.

e-mail: eduarda_ramiao@hotmail.com

Hugo Rodrigues-Fernandes. Psicólogo. Região Autónoma da Madeira, Portugal. e-mail: hugorodriguesfernandes.psi@outlook.com

Sofia D. Quesado. Psicóloga, Instituto de Formação e Transportes (IFT). Viana do Castelo, Portugal. e-mail:ngfia_21@hotmail.com 
Poucos são os estudos que têm procurado analisar as relações entre as variáveis aferidas pela Perceção de Apoio Social (PAS) com as do Bem-Estar Psicológico (BEP) em amostras de adolescentes portugueses. Porém, ao longo dos tempos as conclusões dos estudos sobre comportamento humano tendem a remeter para a necessidade de se atender a fatores não só biológicos, mas também psicológicos, sociais e culturais, uma vez estes domínio são reconhecidamente aceites pela comunidade científica como integradores e mediadores da ontogénese humana (Jackson, Cumming, Drenowatz, Standage, Sherar, \& Malina, 2013; Reed, Bell, \& Edwards, 2011; Rees, Sabia, \& Argys, 2009; Siddall, Huebner, \& Jiang, 2013; Vasconcelos-Raposo, 1993; Vaux, 1988). Neste enlace, a adolescência constitui um período de transformações e integrações significativas, importantes para a diferenciação do indivíduo na fase adulta. Ser adolescente passa por atravessar uma fase de construção de identidade e de descoberta de si, associada a uma natural instabilidade emocional e a alterações físicas e cognitivas (Cavalcante, Alves, \& Barroso, 2008; Fernandes, Vasconcelos-Raposo, Bertelli, \& Almeida, 2011; Rees et al., 2009). Deste modo, deve compreender-se até que ponto a perceção de apoio, por parte do adolescente, se associa às diversas questões a respeito da saúde e satisfação com a vida (Rawana, 2013; Siddal et al., 2013), onde o BEP exerce igualmente uma significativa influência (Freire \& Tavares, 2011). Para Tomé, Camacho, Matos e Diniz (2011), manter uma comunicação satisfatória, quer com os familiares, sobretudo com os pais, quer com os pares, pode ser facilitador de um relacionamento positivo com ambos, levando a uma maior sensação de bem-estar e satisfação com a vida.

Num apoio fornecido às teorias do desenvolvimento, dentro do seu campo de ação, estudos centrados na visão da Psicologia Positiva têm procurado descentrar o foco no sofri- mento e na psicopatologia, como vertentes únicas da ação da Psicologia Clínica. Com o tempo, passou-se a desenvolver uma atitude focada na prevenção e promoção da saúde, da felicidade e do bem-estar, procurando olhar às qualidades e características positivas envoltas nos fenómenos passíveis de observação ou intervenção, com apelos a encorajar modelos psicológicos mais positivos que potenciem o desenvolvimento humano (Selligman \& Csikszentmihalyi, 2000). Neste prisma temos o Bem-Estar Subjetivo e o BEP como constructos alvo de atenção empírica e clínica em Psicologia Positiva (e.g. Fernandes, Vasconcelos-Raposo, Pereira, Ramalho, \& Oliveira, 2009; Freire \& Tavares, 2011; Liu \& Yu, 2013; Selligman \& Csikszentmihalyi, 2000; Siddall et al., 2013; Zeidner \& Olnick-Shemesh, 2010). No que concerne ao BEP, este tem sido crescentemente explorado no domínio empírico, desde o final do século passado (Ryff, 1989). De natureza eudamónica, um movimento Aristotélico que sustenta a visão de desenvolvimento como processo de florescimento e realização do ser humano (Fernandes \& Vasconcelos-Raposo, 2008), o BEP demarca-se do Bem-estar Subjetivo pelo facto de, ao contrário do segundo, este associar-se a uma explicação semântica mais minuciosa, decomposta em seis dimensões do funcionamento psicológico, nomeadamente na autonomia, do domínio do meio, do crescimento pessoal, das relações positivas com os outros, dos objetivos na vida e aceitação de si (Fernandes \& Vasconcelos, 2008; Freire \& Tavares, 2011; Ryff, 1989).

Por outro lado, a PAS tem vindo a revelar-se um constructo pertinente na pesquisa empírica associada à adolescência, desde os seus primeiros estudos no (Antunes \& Fontaine, 1994; Antunes \& Fontaine, 2005; Rawana, 2013; Vaux, 1988). A PAS diz respeito às funções levadas a cabo por grupos ou pessoas significativamente importantes na vida do adolescente, tendo em conta os diversos contextos onde este se insere (e.g. 
família, escola). A perceção de apoio resulta do que o sujeito acha sobre o modo como o estimam e do interesse que sente ser manifestado por ele, da parte dos demais agentes de socialização com quem mantém relação (Antunes \& Fontaine, 1994; Vaux, 1988). Segundo Antunes e Fontaine (2005), o apoio social parece relacionar-se com o ajustamento psicossocial do adolescente, bem como, com o processo da sua autoestima e identidade. Questões adjacentes ao apoio social e as relações sociais positivas, têm sido progressivamente estudadas e indicadas como fatores importantes na contribuição para a saúde e bem-estar (Reblin \& Uchino, 2008; Reed et al., 2011; Tomé et al., 2011). Porém, ao nível do BEP, poucas têm sido as relações estudadas decorrentes dos efeitos que a PAS possa ter, fundamentalmente no que ao contexto português e em amostra de adolescentes. $\mathrm{Na}$ sua generalidade, a PAS parece relacionar-se positivamente com o BEP (Liu \& Yu, 2013), mas mantêm-se em aberto questões em aberto relativamente a dimensões específicas e como estas se relacionam com as do BEP, nomeadamente: o apoio dos pares, das famílias, dos professores, figuras bem presentes na vida do adolescente.

Outra noção patente é que as atitudes e comportamentos dos adolescentes associam-se ao espaço cultural dos grupos onde se inserem, que permitem, em maior ou menor grau, a partilha de ideias e experiências (Cavalcante et al., 2008; Tomé et al., 2011). Em alguns contextos de estudo, como por exemplo o escolar, os dados têm apontado para a importância do apoio dos pares, uma vez que estes parecem prevenir questões ligadas à saúde, desenvolvimento positivo de competências sociais, satisfação global com a vida e aprendizagem (Mota \& Matos, 2010; Shahar, Cohen, Grogan, Barile, \& Henrich, 2009). Siddall et al. (2013) concluíram que os pares de facto exercem uma influência significativa no que diz respeito à satisfação com a vida dos adolescentes, sobretudo nas raparigas. Por outro lado, nos seus achados, não existiram diferenças significativas sobre a relação professor-aluno e a satisfação escolar. Num estudo longitudinal que visou analisar o apoio emocional de professores aos alunos, Pössel, Rudasill, Sawyer, Spence, e Bjerg (2013) concluíram que a perceção de apoio se mostra inversamente associada a sintomas depressivos em alunos com níveis de stresse e depressão médios e elevados. Contudo, os autores apontaram a existência de efeitos iatrogénicos em alunos com baixos níveis de stresse e depressão.

Segundo Fernandes et al. (2011), o BEP relaciona-se positivamente com a satisfação escolar. Sousa, Maia, e Vasconcelos-Raposo (2012) referem que o contexto escolar simboliza um ponto de referência para o desenvolvimento das várias dimensões do BEP no adolescente, sendo que modelos preditores desenvolvidos das investigações empíricas nesta área podem auxiliar o desenvolvimento de políticas educativas. Partindo deste pressuposto, qual o papel da escola no desenvolvimento de competências associadas ao BEP nos adolescentes? Segundo Fernandes et al. (2011) é necessário ainda aprofundar a compreensão dos fenómenos associados à adolescência, tendo por quadro de referência as inúmeras realidades sociais que se consubstanciam em distintos domínios de realização de vida dos adolescentes. Partindo do pressuposto anterior, estudos precedentes como o de Sousa et al. (2012) ou Fernandes e Vasconcelos-Raposo (2008) abordaram as práticas religiosas como fator social e cultural que pode influenciar o BEP. Ora, tendo em conta que a prática religiosa é uma atividade cultural da sociedade portuguesa (Fernandes \& Vasconcelos-Raposo, 2008) qual será o impacto, não só desta mas de outras Atividades de Tempos Livres (ATL) ao nível do BEP e PAS por parte dos adolescentes? Neste seguimento, parece conveniente questionar a influência das ATL 
ao nível do BEP e PAS, para verificar passiveis preditores de apoio e BEP em tais contextos, já que em alguns casos são parte integrante na vida dos jovens.

Referente à família e consequente apoio familiar, Fernandes et al. (2011) identificaram relações positivas quanto à qualidade de relações entre pais, filhos e a satisfação escolar, tendo esta última demonstrado efeitos positivos ao nível do BEP. Algumas evidências levantam a hipótese de que não é a constituição familiar que determina a qualidade dos aspetos associados à adaptação psicossocial positiva do adolescente, mas sim a qualidade dos vínculos mantidos nos núcleos estruturais da família (Fernandes et al., 2011; Mombelli, Costa, Marcon, \& Moura, 2011; Shahimi, Heaven, \& Ciarrochi, 2013). Segundo Fernandes e Vasconcelos-Raposo (2008), a relação entre a estrutura e a dinâmica familiar deve continuar a ser alvo de cogitações por parte dos investigadores. Alguns estudos realçam a importância da família na satisfação com a vida, manifestada pelo adolescente, dado que o apoio identificado revelou relações positivas com a satisfação dos jovens (Siddall et al., 2013; Tomé et al., 2011). Numa pesquisa longitudinal (Shahimi et al., 2013) concluiu-se que os estilos parentais são importantes para a determinação do BEP no adolescente. Os autores verificaram que estilos permissivos potenciaram uma diminuição da esperança, aumento da autoestima e do psicoticismo. Por sua vez, estilos parentais responsivos apareceram associados a aumentos da esperança, autoestima e diminuição do psicoticismo. No caso dos estilos autoritários, estes mostraram indicadores que se relacionaram negativamente com a autoestima dos adolescentes. Fernandes e Vasconcelos-Raposo (2008) veiculam que a independência do adolescente deve associar-se a certos níveis de autonomia (e.g. física, económica, de valores e aptidões), associada a exigências colocadas pelos familiares e demais agentes sociais.
A presente investigação pretende contribuir para o entendimento holístico em torno do BEP com enfoque na possível moderação causal das diversas dimensões da PAS. Dada a escassez empírica neste âmbito, sobretudo ao nível do panorama português. Assim, urge a necessidade de compreender determinadas questões como, por exemplo, em que medida a PAS pode predizer efeitos no modo como se processa o florescimento humano, numa fase em que as relações sociais tendem a integrar-se além das fronteiras relacionais do seio familiar? Que impacto pode ter tanto a perceção do apoio familiar, quanto essa maior envolvência no contexto social mais alargado, sobretudo face ao investimento relacional com o grupo de pares? Os contextos de interação entre pares podem predizer diferenças? Por outro lado, segundo vários autores (e.g Antunes \& Fontaine, 1994; Fernandes \& Vasconcelos, 2008; Sousa et al., 2012), é útil prolongar os estudos no que concerne à adolescência, de modo a adquirir um entendimento mais pormenorizado, pois esta é uma fase marcada por padrões culturais específicos, com efeitos que variam no tempo e contextos, constituindo um desafio constante para a investigação.

Face ao exposto, o objetivo principal do presente trabalho visa compreender os efeitos da PAS sobre o BEP em adolescentes portugueses. Deste modo, os objetivos específicos passam por efetuar comparações das dimensões do BEP por níveis de PAS (menores, médios e elevados), no que se refere às dimensões do constructo, nomeadamente a perceção de apoio emocional dos amigos, da família, dos professores e perceção de apoio emocional geral. É ainda propósito específico comparar o BEP e PAS por sexo, ATL e Tipo de Família (TF).

\section{MÉTODOS}

Este estudo é de caráter transversal quantitativo, quasi-experimental, nomotético e comparativo. A transversalidade do estudo refere-se ao facto dos dados terem sido retirados da 
mostra num único momento. O seu caráter quantitativo e quasi-experimental refere-se à componente estatística, numérica e à tipificação da investigação para contexto clínico, sendo que não recorre a uma distribuição aleatória da amostra e que não existe controlo das variáveis independentes. A sua natureza nomotética justifica-se tendo em conta a dimensão da amostra e o cariz comparativo pela análise dos efeitos dos grupos estudados.

\section{Amostra}

A amostra foi constituída com recurso a um processo de amostragem de conveniência, sendo que não é representativa da população geral e os dados não devem ser generalizados ao total da população com as características da nossa amostra. As escolhas institucionais foram deliberadamente constituídas sem recurso a técnicas aleatórias de amostragem. A amostra inicial foi composta por 314 adolescentes, sendo que face a desistências e critérios de seleção esta foi reduzida para 283 estudantes do $3^{\circ}$ ciclo do ensino básico e do ensino secundário, de um estabelecimento de ensino localizado no norte interior de Portugal. Definimos como critérios de exclusão alunos de nacionalidade não portuguesa, por só pretendermos analisar o fenómeno em adolescentes portugueses e para despistar possíveis vieses de interpretação semântica dos instrumentos. Outro critério de exclusão passou por não incluir alunos com menos de 12 anos e mais de 18 anos, uma vez que o número de casos era reduzido (ver Quadro 1). Deste modo, a idade dos participantes variou entre os $12 \mathrm{e}$ os 17 anos (ver caracterização da amostra no Quadro 1.

Não se verificou qualquer mortalidade na amostra nem se constatou a existência de qualquer evento que pudesse interferir com o preenchimento e participação voluntário dos estudantes.

\section{Instrumentos}

A caracterização da amostra fez-se a partir de um questionário sociodemográfico onde começamos por levantar questões quanto ao sexo e à idade. Reuniu-se ainda informações relativas ao nível de escolaridade, prevalência de reprovações e número de vezes em que estas ocorreram. Também recolhemos informação relativa ao local de residência (rural ou urbana), distrito de residência e nacionalidade. Para verificar a prevalência de consumo de cigarros e álcool suportamo-nos no trabalho de Ciliska e Wilson (1984) de onde adaptamos os itens 8 (tabaco) e 12 (álcool) da escala de estilos de vida FANTASTIC. Relativamente ao consumo de tabaco, a questão prevê a análise entre as categorias mais de 10 cigarros por dia, 1 a 10 cigarros por dia, nenhum no mês passado, nenhum nos últimos seis meses, nenhum nos últimos 5 anos. A fim de despistar a manifestação de consumo e abstinência total foi criada a opção de resposta nunca houve consumo, que constituiu o grupo 1 na categorização dos níveis de consumo. O segundo grupo diz respeito a todos os que fumam ou já fumaram anteriormente. Para o álcool a questão centrou-se no consumo médio por semana, com opções de resposta de mais de 20 doses, 13 a 20 doses, 11-12 doses, 8-10 doses, às quais modificamos a resposta 0-7 doses para a opção 1-7 doses e acrescentamos a possibilidade de escolha de 0 doses, a fim de despistar os adolescentes que não costumam consumir álcool. Para caraterizar a amostra dividiu-se os grupos em adolescentes que consumiam habitualmente e os que não consumiam.

A questão sobre que figuras parentais viviam com os adolescentes, para determinar assim o TF, adveio da proposta de Mano e Costa (2011), sendo que foram criados quatro grupos, em função dos aglomerados familiares identificados. Assim, tivemos o grupo família nuclear (pai, mãe e potencialmente irmãos), família monoparental (pai ou mãe e potencialmente irmãos), famílias reconstituídas (pai ou 
Quadro 1

Caracterização da amostra.

\begin{tabular}{|c|c|c|c|}
\hline & & $N$ & $\%$ \\
\hline Amostra Global & & 283 & 100 \\
\hline \multicolumn{4}{|l|}{ Sexo } \\
\hline & Feminino & 149 & 52.7 \\
\hline & Masculino & 134 & 47.3 \\
\hline \multicolumn{4}{|l|}{ Idade } \\
\hline & $12-13$ & 77 & 27.2 \\
\hline & $14-15$ & 150 & 53.0 \\
\hline & $16-17$ & 56 & 19.8 \\
\hline \multicolumn{4}{|l|}{ Reprovações } \\
\hline & Sim & 29 & 10.2 \\
\hline & Não & 254 & 89.8 \\
\hline \multicolumn{4}{|c|}{ Local de Residência } \\
\hline & Urbano & 194 & 68.6 \\
\hline & Rural & 89 & 31.4 \\
\hline \multicolumn{4}{|c|}{ Hábitos de Consumo de Substâncias } \\
\hline & Fuma/Já fumou & 44 & 15.5 \\
\hline & Nunca fumou & 239 & 84.5 \\
\hline & Consome álcool & 34 & 11.7 \\
\hline & Não Consome álcool & 249 & 88.3 \\
\hline \multicolumn{4}{|c|}{ Agregado Familiar } \\
\hline & Família nucleares & 208 & 73.5 \\
\hline & Família monoparentais & 29 & 9.9 \\
\hline & Família reconstituídas & 15 & 5.3 \\
\hline & Família alargadas & 31 & 11.3 \\
\hline \multicolumn{4}{|c|}{ Empregabilidade da Mãe } \\
\hline & Sim & 249 & 88 \\
\hline & Não & 34 & 12 \\
\hline \multicolumn{4}{|c|}{ Empregabilidade do Pai } \\
\hline & Sim & 250 & 88.3 \\
\hline & Não & 33 & 11.7 \\
\hline \multicolumn{4}{|c|}{ Prática de Atividades Tempos Livres } \\
\hline & Desporto/ Exercício Físico & 147 & 51.9 \\
\hline & Outras Atividades & 58 & 20.1 \\
\hline & Não Pratica & 78 & 28.0 \\
\hline
\end{tabular}

mãe, padrasto ou madrasta e irmãos) e famílias alargadas (para além das figuras primárias do aglomerado familiar constavam outras figuras como avós, tios, primos, por exemplo). Foi também formulada uma questão para compreender a situação de empregabilidade atual dos pais dos adolescentes. Por último, para a prática de ATL foi criado um item com quatro categorias subjacentes a atividades desportivas/ atividade física, atividades artísticas, atividades religiosas e outras, sendo que os grupos foram criados a partir das caracterizações dos não praticantes, e praticantes na área do desporto/ exercício físico e os restantes 
incluídos na categoria de praticantes de outras atividades ou mais de duas atividades.

Para avaliar o BEP foi utilizada a versão portuguesa da escala BEP, versão reduzida a 30 itens, concebida por Fernandes, Vasconcelos-Raposo e Teixeira (2010). Esta versão derivou dos trabalhos iniciais levados a cabo por Ryff (1989). Trata-se de uma escala de auto-relato, organizada segundo uma escala tipo Likert de cinco pontos em que 1 corresponde à avaliação discordo plenamente e 5 corresponde terminologicamente a concordo plenamente. A escala avalia o BEP em seis dimensões, sendo estas Autonomia (itens 1; 7; $3 ; 19 ; 25)$, Domínio do meio $(2 ; 8 ; 14 ; 20 ; 26)$, Crescimento pessoal (itens $3 ; 9 ; 15 ; 21 ; 27$ ), Relações positivas (itens $4 ; 10 ; 16 ; 22 ; 28$ ), Objetivos de Vida (itens $5 ; 11 ; 17 ; 23 ; 29$ ) e Aceitação de Si (itens $6 ; 12 ; 18 ; 24 ; 30$ ). Deve ser realizada a inversão dos itens 2,5 e 19, uma vez que são formulados na negativa. O Alpha de Cronbach da versão original oscilou entre .79 e .83 (Ryff, 1989). O Alpha de Cronbach da proposta de Fernandes e colaboradores (2010) variou nos valores de alpha entre .85 e .90 .

As dimensões do apoio social foram mensuradas a partir da Social Support Appraisals Scale, instrumento proposto por Vaux (1988) e adaptado para a população portuguesa por Antunes e Fontaine (1994). A versão original da escala (Vaux, 1988) possibilita uma avaliação sobre o apoio social percebido, nas dimensões associadas à perceção de apoio emocional familiar, amigos e ainda o apoio geral. $\mathrm{Na}$ adaptação portuguesa realizada por Antunes e Fontaine (1994) foi acrescentada outra dimensão, referente à perceção de apoio emocional dos professores. A versão portuguesa (Antunes \& Fontaine, 1994) utilizada na presente investigação, organiza-se numa escala de Likert de seis pontos, variando entre (1) discordo totalmente até (6) concordo totalmente e divide-se em quatro subescalas. Essas dizem respeito à perceção de apoio dos amigos (itens $1 ; 8 ; 11 ; 14 ; 17 ; 24 ; 27)$, perceção de apoio da família (itens $3 ; 6 ; 9 ; 16 ; 19 ; 22 ; 25 ; 30$ ), perceção de apoio dos professores (itens $2 ; 5 ; 12 ; 15 ; 18 ; 21$; 28) e por último a perceção de apoio em geral (itens $4 ; 7 ; 10 ; 13 ; 20 ; 23 ; 26 ; 29)$. No que se refere à inversão de itens esta deve ser levada a cabo nos itens $4 ; 11 ; 18 ; 22 ; 28 ; 29$ e 30. Quanto às qualidades psicométricas, num teste à consistência interna, num estudo de Antunes e Fontaine (2005), a escala apresentou qualidades psicométricas adequadas tanto na escala global $(\alpha=.91)$ como nas subescalas, apoio dos amigos ( $\alpha=$ $.79)$, apoio da família $(\alpha=.80)$, apoio de professores $(\alpha=.79)$ e apoio geral $(\alpha=.72)$.

\section{Desenho do Estudo}

Para análise do efeito da PAS sobre o BEP, definimos como variáveis independentes a PAS, nas suas 4 dimensões. Nesta fase, foi variável dependente o BEP e as suas 6 dimensões. Para análise da influência das ATL e TF, estas foram determinadas como variáveis independentes, sendo que a PAS passou a ser variável dependente, juntando-se ao BEP. O nosso estudo resulta de um desenho experimental (PAS: PAS total, apoio dos amigos, apoio familiar, apoio dos professores e apoio em geral; Sexo: feminino e masculino ATL: não praticante, praticantes de desporto e praticantes de outras atividades; TF: família nuclear, monoparental, reconstituída e alargada).

\section{Procedimentos}

Após parecer favorável pelo comité de ética e das autoridades escolares, para realização da investigação, foram considerados os alunos cujos encarregados de educação, previamente informados, autorizaram a participação do seu educando no estudo. Os alunos foram também informados sobre os propósitos da pesquisa, sendo que foram clarificadas noções associadas à voluntariedade de participação e confidencialidade dos dados, tendo em conta os procedimentos éticos da investigação em Psicologia. À recolha de dados não esteve associada qualquer recompensa monetária ou de outro cariz aos participantes. A recolha de dados foi reali- 
zada a partir da técnica de testagem em grupo, em contexto sala de aula, onde os professores despenderam algum tempo para a realização da recolha. Entre instruções e recolha dos questionários, as avaliações duraram cerca de quinze minutos. Todas as condições logísticas foram garantidas.

\section{Análise Estatística}

Para tratamento estatístico recorremos ao Statistical Package for the Social Science (SPSS), versão 25. De início, efetuaram-se testes com vista à caracterização descritiva da amostra com base nos indicadores de tendência centrar (média) e dispersão (desvio padrão) do conteúdo analisado. De seguida procedeu-se ao tratamento e cotação dos itens das escalas. Recorrendo as técnicas de triangulação de dados procedeu-se à operacionalização e transformação da PAS em variável categórica. Para tal, submeteram-se as escalas do PAS uma divisão das pontuações em quartis, para assim constituir os grupos que foram designados por índices menores, médios e elevados.

Efetuaram-se também testes Skewness $e$
Kurtosis, para analisar a simetria e achatamento das variáveis dependentes, bem como a análise da confiabilidade a partir do alfa de Cronbach's. Por fim, para testar as hipóteses, efetuaram-se análises multivariadas da variância (MANOVA), com recurso ao teste de Post-Hoc de Bonferroni. Foram ainda tidos em consideração a observação da homogeneidade, e a dimensão dos efeitos $\left(\eta_{\mathrm{p}}^{2}\right)$ e tendo em conta o teste Wilk's Lambda. Determinou-se o nível de confiança a 95\% $(p<.05)$.

\section{RESULTADOS}

Passamos a apresentar os resultados descritivos e de análise da normalidade das escalas utilizadas, assim como o coeficiente de consistência interna de cada um dos instrumentos (ver Quadro 2). Considerando o tamanho da nossa amostra, verificou-se que os coeficientes de assimetria (Skewness) e achatamento (Kurtosis) situaram-se dentro do intervalo previsto para a unidade (-2 a 2 Skewness; -3 a 3 Kurtosis). Com isto, assumiu-se a normalidade da distribuição de dados, dada também a dimensão da amostra avaliada, podendo trata-los a partir de testes paramétricos.

Quadro 2

Análise descritiva da assimetria, achatamento e consistência interna (Skewness, Kurtosis e a de Cronbach).

\begin{tabular}{cccccc}
\hline & Média & Desvio-padrão & Skewness & Kurtosis & $\alpha$ Cronbach \\
\hline BEP (G) & 118.81 & 13.93 & -.875 & .895 & .87 \\
\hline A & 19.67 & 3.15 & -.442 & .069 & .46 \\
DM & 18.24 & 2.85 & -.609 & .507 & .47 \\
CP & 22.27 & 2.63 & -1.271 & 2.322 & .69 \\
RP & 21.03 & 3.16 & -1.196 & 2.589 & .67 \\
OV & 18.17 & 3.89 & -.476 & -.212 & .69 \\
AS & 19.44 & 4.26 & -1.101 & 1.194 & .84 \\
PAS (G) & 144.3 & 18.05 & -.903 & 1.181 & .91 \\
\hline AEA & 35.71 & 5.42 & -1.103 & 1.436 & .87 \\
AEF & 42.80 & 5.91 & -1.561 & 2.269 & .85 \\
AEP & 28.48 & 6.41 & -.504 & .345 & .82 \\
AEG & 37.36 & 6.36 & -1.017 & 2.219 & .81 \\
\hline
\end{tabular}

Nota: BEP (G) - Bem-Estar Psicológico Global; A - Autonomia DM - Domínio do Meio; CP - Crescimento Pessoal; RP - Relações Positivas; OV - Objetivos de Vida; AS- Aceitação de Si; PAS (G)- Perceção de Apoio Social Global; AEAApoio Emocional dos Amigos; AEF- Apoio Emocional da Família; AEP- Apoio Emocional dos Professores; AEG- Apoio Emocional Geral. 
A partir de uma MANOVA comparámos as dimensões do BEP pelos grupos referentes aos valores globais da escala de PAS (Quadro 3), sendo que as diferenças entre grupos se realizaram com recurso ao teste Post Hoc (Bonferroni). Nesta comparação identificámos efeitos significativos, quer na comparação dos valores globais do BEP, bem como na análise comparativa pelas restantes subescalas $\left[F_{(12,550)}=\right.$ 14.994; $p=<.001$, Wilk's $\lambda=.568$ ]. Quanto à comparação por grupos observa-se que nas dimensões Autonomia e Crescimento Pessoal existem diferenças significativas quanto ao grupo de menores índices de apoio social face aos restantes grupos sem que existam dife- renças entre grupo de índices médios e elevados de apoio social apresentados pela amostra $\left[F_{(12,550)}=14.994 ; p=<.001\right.$, Wilk's $\left.\lambda=.568\right]$. Tais comparações são explicadas à luz de um efeito moderado para as dimensões Autonomia $\left(\eta_{\mathrm{p}}^{2}=.057\right)$ e Crescimento Pessoal $\left(\eta_{\mathrm{p}}^{2}=\right.$ .121). Para as restantes comparações os valores apresentam uma tendência crescente das variáveis dependentes à medida que os valores nas independentes aumentam $\left[F_{(12,550)}=14.994\right.$; $p=<.001$, Wilk's $\lambda=.568]$, com efeito forte para o Domínio do Meio $\left(\eta^{2}=.202\right)$, Objetivos de Vida $\left(\eta_{\mathrm{p}}^{2}=.178\right)$, o Bem-Estar Psicológico Global $\left(\eta_{p}^{2}=.375\right)$, Relações Positivas $\left(\eta_{p}^{2}=\right.$ .221), e Aceitação de Si $\left(\eta_{p}^{2}=.341\right)$.

Quadro 3

Comparação das dimensões do BEP por nível da PAS (MANOVA).

\begin{tabular}{|c|c|c|c|c|c|c|c|}
\hline & $\begin{array}{c}\text { MIPAS } \\
(n=71) \\
M \pm D P\end{array}$ & $\begin{array}{c}\text { IMPAS } \\
(n=144) \\
\text { M } \pm \text { DP }\end{array}$ & $\begin{array}{c}\text { IEPAS } \\
(n=68) \\
M \pm D P\end{array}$ & $F$ & $\mathrm{P}$ & $\eta_{p}^{2}$ & $\begin{array}{c}\neq \text { Sig. entre grupos } \\
\text { Post Hoc }\end{array}$ \\
\hline $\operatorname{BEP}(\mathrm{G})$ & $105.14 \pm 14.24$ & $120.90 \pm 10.21$ & $128.68 \pm 8.74$ & 83.902 & $<.001$ & .375 & MIPAS < IMPAS <IEPAS \\
\hline A & $18.49 \pm 3.15$ & $19.81 \pm 3.19$ & $20.60 \pm 2.70$ & 8.487 & $<.001$ & .057 & MIPAS <IMPAS; IEPAS \\
\hline $\mathrm{DM}$ & $16.14 \pm 2.86$ & $18.61 \pm 2.59$ & $19.63 \pm 2.12$ & 35.525 & $<.001$ & .202 & MIPAS < IMPAS < IEPAS \\
\hline $\mathrm{CP}$ & $20.76 \pm 3.17$ & $22.56 \pm 2.26$ & $23.22 \pm 2.05$ & 19.238 & $<.001$ & .121 & MIPAS <IMPAS; IEPAS \\
\hline RP & $18.80 \pm 3.55$ & $21.20 \pm 2.77$ & $23.00 \pm 1.77$ & 39.720 & $<.001$ & .221 & MIPAS < IMPAS < IEPAS \\
\hline OV & $15.59 \pm 4.07$ & $18.49 \pm 3.47$ & $20.16 \pm 3.05$ & 30.253 & $<.001$ & .178 & MIPAS < IMPAS < IEPAS \\
\hline AS & $15.35 \pm 4.88$ & $20.22 \pm 2.86$ & $22.06 \pm 2.81$ & 72.306 & $<.001$ & .341 & MIPAS $<$ IMPAS $<$ IEPAS \\
\hline
\end{tabular}

Nota: Variáveis independentes: MIPAS- Menores Índices de Perceção de Apoio Social, IMPAS- Índices Médios de Perceção de Apoio Social, IEPAS- Índices Elevados de Perceção de Apoio Emocional; Variáveis dependentes: BEP (G) Bem-Estar Psicológico Global; A- Autonomia; DM- Domínio do Meio; CP- Crescimento Pessoal; RP- Relações Positivas; OV- Objetivos de Vida; AS- Aceitação de Si.

A partir da MANOVA procedeu-se à comparação das dimensões do BEP pelos grupos apresentados na dimensão de Apoio Emocional dos Amigos (Quadro 4), sendo que as diferenças entre grupos se realizaram com recurso ao teste Post Hoc (Bonferroni). Os resultados não mostraram efeitos nas variáveis dependentes, quer na comparação dos valores globais do BEP, quer em nas dimensões da escala, com exceção da autonomia com um efeito fraco $\left[F_{(12,550)}\right.$ $=14.935 ; p=.055$, Wilk's $\lambda=.569, \eta_{p}^{2}=$ .020]. Todos os restantes apresentaram efeitos moderados ou forte. Quanto à comparação por grupos da escala global de BEP observam- -se diferenças significativas com efeito forte $\left[F_{(12,550)}=14.935 ; p=<.001\right.$, Wilk's $\lambda=.569$, $\left.\eta_{\mathrm{p}}^{2}=.232\right]$, e no que se refere à subescalas constatamos efeitos moderados na variável domínio do meio e objetivos de vida. Nos restantes obtiveram-se efeitos fortes com particular destaque para a variável relações positivas. No que concerne ao Domínio do Meio, indivíduos com menores índices de apoio dos amigos evidenciam um menor score neste constructo comparativamente aos adolescentes dos outros dois grupos, tendo, este efeito, um valor moderado $\left[F_{(12,550)}=14.935 ; p=<.001\right.$, Wilk's $\left.\lambda=.569, \eta_{\mathrm{p}}^{2}=.085\right]$. No Crescimento Pessoal 
mantém-se a tendência anterior sendo que os valores predizem menores pontuações para os adolescentes com menores índices de apoio dos amigos comparativamente aos outros dois grupos, com efeito forte $\left[F_{(12,550)}=14.935 ; p=\right.$ $<.001$, Wilk's $\left.\lambda=.569, \eta_{\mathrm{p}}^{2}=.144\right]$. Para as Relações Positivas a análise efetuada exprime a existência de diferenças significativas na comparação por grupos $\left[F_{(12,550)}=14.935 ; p=\right.$ $<.001$, Wilk's $\lambda=.569$ ], sendo que as diferenças significativas apontam que à medida que aumenta o grau de apoio percebido pelos adolescentes da parte dos amigos, aumenta também os níveis deste constructo, manifestando este efeito um valor forte $\left(\eta_{p}^{2}=.382\right)$.
Face aos Objetivos de Vida a amostra apresenta diferenças significativas na medida em que indivíduos com menores índices de apoio dos amigos apresentam um valor mais reduzido da média comparativamente aos grupos de adolescentes com índices médios e elevados de apoio dos amigos, com um efeito moderado $\left[F_{(12,550)}=14.935 ; p=<.001\right.$, Wilk's $\lambda=.569$, $\left.\eta_{p}^{2}=.056\right]$. No constructo Aceitação de Si, os estudantes com menores índices de apoio dos amigos seguem a mesma tendência que no constructo anterior, tendo este efeito um valor forte $\left[F_{(12,550)}=14.935 ; p=<.001\right.$, Wilk's $\lambda=$ $\left..569, \eta_{\mathrm{p}}^{2}=.153\right]$.

Quadro 4

Comparação das dimensões do BEP por nível de perceção do adolescente face ao apoio dos amigos (MANOVA).

\begin{tabular}{|c|c|c|c|c|c|c|c|}
\hline & $\begin{array}{c}\text { MIAEA } \\
(n=75) \\
\text { M } \pm \text { DP }\end{array}$ & $\begin{array}{c}\text { IMSEA } \\
(n=149) \\
\text { M } \pm \mathrm{DP}\end{array}$ & $\begin{array}{c}\text { IESEA } \\
(n=59) \\
M \pm D P\end{array}$ & $F$ & $\mathrm{P}$ & $\eta_{p}^{2}$ & $\begin{array}{c}\neq \text { Sig. entre grupos } \\
\text { Post Hoc }\end{array}$ \\
\hline BEP (G) & $107.93 \pm 16.04$ & $121.66 \pm 10.46$ & $125.46 \pm 10.84$ & 42.243 & $<.001$ & .232 & MSIEA <IMSEA;IESEEA \\
\hline A & $18.92 \pm 3.13$ & $19.91 \pm 3.03$ & $20.00 \pm 3.37$ & 2.927 & .055 & .020 & n.s. \\
\hline $\mathrm{DM}$ & $18.87 \pm 3.21$ & $18.65 \pm 2.46$ & $18.93 \pm 2.76$ & 12.991 & $<.001$ & .085 & MISEA < IMSEA; IESEA \\
\hline $\mathrm{CP}$ & $20.64 \pm 3.16$ & $22.72 \pm 2.22$ & $23.20 \pm 1.88$ & 23.537 & $<.001$ & .144 & MISEA <IMSA; IESA \\
\hline RP & $18.15 \pm 3.28$ & $21.42 \pm 2.31$ & $23.71 \pm 1.62$ & 86.367 & $<.001$ & .382 & MISEA $<$ IMSEA $<$ IESEA \\
\hline OV & $16.64 \pm 4.36$ & $18.75 \pm 3.35$ & $18.63 \pm 4.05$ & 8.297 & $<.001$ & .056 & MISEA < IMSEA; IESEA \\
\hline AS & $16.72 \pm 5.34$ & $20.20 \pm 3.10$ & $20.98 \pm 3.73$ & 25.275 & $<.001$ & .153 & MISEA < IMSEA; IESEA \\
\hline
\end{tabular}

Nota: Variáveis independentes: MIAEA- Menores Índices de Apoio Emocional dos Amigos, IMAEA- Índices Médios de Apoio Emocional dos Amigos, IEAEA- Índices Elevados de Apoio Emocional dos Amigos; Variáveis dependentes: BEP (G) - Bem-Estar Psicológico Global; A - Autonomia; DM - Domínio do Meio; CP - Crescimento Pessoal; RP - Relações Positivas; OV - Objetivos de Vida; AS - Aceitação de Si.

Numa outra MANOVA procedeu-se à comparação das dimensões de BEP por grupos dos índices de Apoio Emocional da Família (ver Quadro 5. Para identificar as diferenças entre os grupos realizou-se um teste Post Hoc (Bonferroni). Assim, para o BEP global as comparações indicam que existem diferenças significativas entre os três grupos, sendo que à medida que aumentam os valores do apoio da família tendem a aumentar os níveis de BEP com um valor de efeito forte $\left[F_{(12,550)}=8.488\right.$; $p=<.001$, Wilk's $\left.\lambda=.712, \eta_{\mathrm{p}}^{2}=.254\right]$. Para a Autonomia, estudantes enquadrados no grupo com menores índices de apoio da família apre- sentam menores valores deste constructo na comparação com os indivíduos dos outros dois grupos, sendo este valor de efeito moderado $\left[F_{(12,550)}=8.488 ; p=.001\right.$, Wilk's $\lambda=.712$, $\left.\eta_{\mathrm{p}}^{2}=.052\right]$. Quanto ao Domínio do Meio, a comparação dita diferenças significativas, explicando que face ao aumento do apoio da família, maior a pontuação neste constructo com um score de efeito forte $\left[F_{(12,550)}=8.488\right.$; $p=<.001$, Wilk's $\left.\lambda=.712, \eta_{\mathrm{p}}^{2}=.183\right]$. Nas restantes dimensões, as comparações identificam que os adolescentes com índices menores de perceção de apoio dos seus familiares apresentam valores mais baixos para as 
restantes dimensões de BEP ao mesmo grau de significância $\left[F_{(12,550)}=8.488 ; p=<.001\right.$, Wilk's $\lambda=.712]$. Para quase todas as dimensões o efeito da comparação é moderado
(Crescimento Pessoal- $\eta_{\mathrm{p}}^{2}=.095$; Relações Positivas $\eta_{\mathrm{p}}^{2}=.118$; Objetivos de Vida $\eta_{\mathrm{p}}^{2}=$ .099), à exceção da Aceitação de Si manifestou um efeito forte $\left(\eta_{\mathrm{p}}^{2}=.216\right)$.

Quadro 5

Comparação das dimensões do BEP pelo nível de perceção do adolescente face ao apoio da família (MANOVA).

\begin{tabular}{cccccccc}
\hline & $\begin{array}{c}\text { MIAEF } \\
(n=75) \\
\mathrm{M} \pm \mathrm{DP}\end{array}$ & $\begin{array}{c}\text { IMAEF } \\
(n=150) \\
\mathrm{M} \pm \mathrm{DP}\end{array}$ & $\begin{array}{c}\text { IEAEF } \\
(n=58) \\
\mathrm{M} \pm \mathrm{DP}\end{array}$ & $F$ & $\mathrm{P}$ & $\eta^{2}{ }^{2}$ & $\neq$ Sig. entre grupos \\
\hline BEP (G) & $107.72 \pm 15.24$ & $121.23 \pm 10.97$ & $126.90 \pm 10.02$ & 47.640 & $<.001$ & .254 & MIAEF < IMAEF < IEAEF \\
\hline A & $18.53 \pm 3.25$ & $19.92 \pm 2.90$ & $20.48 \pm 3.32$ & 7.615 & $<.001$ & .052 & MIAEF < IMAEF; IEAEF \\
DM & $16.35 \pm 2.94$ & $18.60 \pm 2.50$ & $19.24 \pm 2.85$ & 31.269 & $<.001$ & .183 & MIAEF <IMAEF <IEAEF \\
CP & $20.95 \pm 3.14$ & $22.63 \pm 2.28$ & $23.05 \pm 2.14$ & 14.754 & $<.001$ & .095 & MIAEF <IAEF; IAEF \\
RP & $19.35 \pm 3.66$ & $21.37 \pm 2.65$ & $22.34 \pm 2.79$ & 18.640 & $<.001$ & .118 & MAEF < IMAEF; IEAEF \\
OV & $16.28 \pm 4.13$ & $18.51 \pm 3.49$ & $19.71 \pm 3.66$ & 15.420 & $<.001$ & .099 & MIAEF < IMAEF; IEAEF \\
AS & $16.27 \pm 5.13$ & $20.21 \pm 3.28$ & $21.57 \pm 2.84$ & 38.668 & $<.001$ & .216 & MIAEF < IMAEF; IEAEF \\
\hline
\end{tabular}

Nota: MIAEF - Menores Índices de Apoio Emocional da Família; IMAEF - Índices Médios de Apoio Emocional da Família; IEAEF - Índices Elevados de Apoio Emocional da Família; Variáveis dependentes: BEP (G) - Bem-Estar Psicológico Global; A - Autonomia; DM - Domínio do Meio; CP - Crescimento Pessoal; RP - Relações Positivas; OV Objetivos de Vida; AS - Aceitação de Si.

Para a comparação por grupos da dimensão de Apoio Emocional dos Professores face às dimensões do BEP (ver Quadro 6) recorreu-se, como até aqui, a uma MANOVA. Igualmente, diferenças entre grupos foram identificadas com recurso ao teste Post Hoc (Bonferroni). Deste modo, quanto à comparação por grupos da escala global de BEP observam-se diferenças significativas com efeito moderado $\left[F_{(12,550)}=\right.$ 4.815; $p=<.001$, Wilk's $\left.\lambda=.819, \eta^{2}=.144\right]$, sendo que existem diferenças significativas entre todos os grupos identificando que quanto maior o nível de apoio percebido pelo adolescente referente aos professores, maior o BEP. No que se refere à Autonomia, as comparações apontam para um menor score quanto a este constructo em indivíduos com menores índices de apoio dos professores comparativamente aos grupos de índices médios e elevados de apoio, a um efeito moderado $\left[F_{(12,550)}=4.815\right.$; $p=.001$, Wilk's $\left.\lambda=.819, \eta_{\mathrm{p}}^{2}=.052\right]$. Nesta comparação e aludindo ao Domínio do Meio, indivíduos com índices elevados de apoio dos professores evidenciam maior pontuação neste constructo, comparativamente aos grupos de índices médios e inferiores de perceção de apoio dos professores, a um grau de efeito moderado $\left[F_{(12,550)}=4.815 ; p=<.001\right.$, Wilk's $\lambda=.819$, $\left.\eta_{\mathrm{p}}^{2}=.088\right]$. Perante o Crescimento Pessoal, as diferenças significativas observadas passam-se ao nível da comparação entre o grupo de adolescentes com baixo apoio emocional dos professores e o grupo com índices elevados de apoio, existindo uma maior pontuação neste constructo para os adolescentes do segundo grupo $\left[F_{(12,550)}=4.815 ; p=.021\right.$, Wilk's $\left.\lambda=.819\right] \mathrm{com}$ efeito fraco $\left(\eta_{p}^{2}=.027\right)$. Nas Relações Positivas a análise indica a existência de diferenças significativas na comparação por grupos, sendo que estas apontam para um maior predisposição a desenvolverem relações positivas, adolescentes com maior grau de apoio percebido dos professores, ainda que sob um valor de efeito fraco $[F$ ${ }_{(12,550)}=4.815 ; p=.019$, Wilk's $\lambda=.819, \eta_{p}^{2}=$ $.028]$. Nas duas últimas comparações podemos observar que quanto maior o índice a perceção dos adolescentes face ao apoio dos professores, maior a pontuação ao mesmo grau de signifi- 
cância $\left[F_{(12,550)}=4.815 ; p=<.001\right.$, Wilk's $\lambda=$ .819], com efeito moderado, quer perante os
Objetivos de Vida $\left(\eta_{\mathrm{p}}^{2}=.106\right)$, quer na Aceitação de si $\left(\eta^{2}=.139\right)$.

Quadro 6

Comparação das dimensões do BEP pelo nível de perceção do adolescente face ao apoio dos professores (MANOVA).

\begin{tabular}{|c|c|c|c|c|c|c|c|}
\hline & $\begin{array}{c}\text { MIAEP ( } \\
\mathrm{n}=74) \\
\mathrm{M} \pm \mathrm{DP}\end{array}$ & $\begin{array}{l}\text { IMAEP ( } \\
\mathrm{n}=148) \\
\mathrm{M} \pm \mathrm{DP}\end{array}$ & $\begin{array}{c}\text { IEAEP } \\
(\mathrm{n}=61) \\
\mathrm{M} \pm \mathrm{DP}\end{array}$ & $F$ & $\mathrm{P}$ & $\eta_{p}^{2}$ & $\neq$ Sig. entre grupos \\
\hline BEP (G) & $112.24 \pm 15.61$ & $118.48 \pm 12.50$ & $127.59 \pm 10.05$ & 23.647 & $<.001$ & .144 & MIAEP <IMAEP <IEAEP \\
\hline A & $18.64 \pm 3.59$ & $19.76 \pm 2.96$ & $20.70 \pm 2.69$ & 7.677 & .001 & .052 & MIAEP <IMAEP; IEAEP \\
\hline DM & $17.26 \pm 3.12$ & $18.13 \pm 2.67$ & $19.69 \pm 2.38$ & 13.465 & $<.001$ & .088 & MIAEP; IMAEP <IEAEP \\
\hline $\mathrm{CP}$ & $21.77 \pm 2.93$ & $22.21 \pm 2.54$ & $23.02 \pm 2.32$ & 3.913 & .021 & .027 & $M I A E P<I E A E P$ \\
\hline RP & $20.70 \pm 3.51$ & $20.78 \pm 3.16$ & $22.03 \pm 2.45$ & 4.014 & $.019 *$ & .028 & MIAEP; IMAEP < IEAEP \\
\hline OV & $16.59 \pm 4.30$ & $18.09 \pm 3.66$ & $20.26 \pm 2.86$ & 16.609 & $<.001$ & .106 & MIAEP $<$ IMAEP $<$ IEAEP \\
\hline AS & $17.28 \pm 5.13$ & $19.51 \pm 3.60$ & $21.89 \pm 3.08$ & 22.566 & $<.001$ & .139 & MIAEP < IMAEP < IEAEP \\
\hline
\end{tabular}

Nota: MIAEP - Menores Índices de Emocional dos Professores; IMAEP - Índices Médios de Apoio Emocional dos Professores; IEAEP - Índices Elevados de Apoio Emocional dos Professores; Variáveis dependentes: BEP (G) - BemEstar Psicológico Global; A - Autonomia; DM - Domínio do Meio; CP - Crescimento Pessoal; RP - Relações Positivas; OV - Objetivos de Vida; AS - Aceitação de Si.

Para comparar os níveis de apoio geral percebido pelos adolescentes (ver Quadro 7), recorreu-se, novamente, à MANOVA e ao teste Post Hoc (Bonferroni). Deste modo, quanto à comparação por grupos da escala global de BEP observam-se diferenças significativas com efeito forte $\left[F_{(12,550)}=10.480 ; p=<.001\right.$, Wilk's $\left.\lambda=.662, \eta_{\mathrm{p}}^{2}=.297\right]$, tendo em conta que quanto maior o nível de apoio geral percebido maior a média obtida no BEP global. Para a Autonomia os dados permitem constatar que indivíduos com menores índices de apoio geral manifestam menores índices de autonomia comparativamente aos grupos de adolescentes com índices médios e elevados de apoio geral com um efeito moderado $\left[F_{(12,550)}=10.480 ; p\right.$ $=<.001$, Wilk's $\left.\lambda=.662, \eta_{\mathrm{p}}^{2}=.053\right]$. No que concerne ao Domínio do Meio, as comparações demonstram que existem diferenças significativas nos três grupos evidenciando que quanto maior os índices de apoio maior a capacidade de domínio do meio. Esta razão é explicada perante um valor de efeito forte $\left[F_{(12,550)}=10.480 ; p=\right.$ $<.001$, Wilk's $\left.\lambda=.662, \eta_{p}^{2}=.166\right]$. Face ao Crescimento Pessoal as comparações indicam que os estudantes com menor apoio geral almejam menores pontuações quanto a este constructo, comparativamente aos adolescentes com índices médios e elevados de apoio geral, sob um efeito moderado $\left[F_{(12,550)}=10.480 ; p=\right.$ $<.001$, Wilk's $\left.\lambda=.662, \eta_{p}^{2}=.098\right]$. Nas Relações Positivas existem diferenças significativas na comparação por grupos $\left[F_{(12,550)}=10.480 ; p\right.$ $=<.001$, Wilk's $\lambda=.662]$, sendo que as diferenças significativas apontam que à medida que aumenta o grau de apoio geral, aumentam também os níveis do constructo, manifestando este efeito um valor forte $\left(\eta_{p}^{2}=.143\right)$. Quanto aos Objetivos de Vida a amostra apresenta diferenças significativas predizendo um aumento substancial das pontuações no constructo, conforme maior o índice de apoio geral apresentado por cada grupo, com um efeito moderado $\left[F_{(12,550)}=10.480 ; p=<.001\right.$, Wilk's $\lambda=$ $\left..662, \eta_{p}^{2}=.138\right]$. Para o constructo Aceitação de $\mathrm{Si}$, a tendência comparativa mantém-se igual face à do constructo anterior, tendo um efeito significativo de valor forte $\left[F_{(12,550)}=10.480 ; p\right.$ $=<.001$, Wilk's $\left.\lambda=.662, \eta_{\mathrm{p}}^{2}=.283\right]$. 
Quadro 7

Comparação das dimensões do BEP pelo nível de perceção do adolescente face ao apoio emocional geral (MANOVA).

\begin{tabular}{|c|c|c|c|c|c|c|c|}
\hline & $\begin{array}{c}\text { MIAEG } \\
(n=80) \\
\text { M } \pm \text { DP }\end{array}$ & $\begin{array}{c}\text { IMAEG } \\
(n=148) \\
M \pm D P\end{array}$ & $\begin{array}{c}\text { IEAEG } \\
(n=55) \\
M \pm D P\end{array}$ & $F$ & $\mathrm{P}$ & $\eta_{p}^{2}$ & $\neq$ Sig. entre grupos \\
\hline $\operatorname{BEP}(\mathrm{G})$ & $107.42 \pm 15.24$ & $121.48 \pm 10.8$ & $128.20 \pm 9.80$ & 59.231 & $<.001$ & .297 & MIAEG <IMAEG <IEAEG \\
\hline $\mathrm{A}$ & $18.54 \pm 3.11$ & $20.00 \pm 2.972$ & $20.42 \pm 3.316$ & 7.889 & $<.001$ & .053 & MIAEG <IMAEG; IEAEG \\
\hline $\mathrm{DM}$ & $16.49 \pm 3.14$ & $18.66 \pm 2.30$ & $19.65 \pm 2.57$ & 27.892 & $<.001$ & .166 & $M I A E G<I M A E G<I E A E G$ \\
\hline $\mathrm{CP}$ & $21.01 \pm 3.19$ & $22.60 \pm 2.25$ & $23.20 \pm 1.98$ & 15.146 & $<.001$ & .098 & MIAEG < IMAEG; IEAEG \\
\hline RP & $19.31 \pm 3.43$ & $21.34 \pm 2.85$ & $22.69 \pm 2.27$ & 23.428 & $<.001$ & .143 & MIAEG < IMAEG < IEAEG \\
\hline OV & $16.09 \pm 4.23$ & $18.54 \pm 3.32$ & $20.18 \pm 3.43$ & 22.506 & $<.001$ & .138 & MIAEG < IMAEG < IEAEG \\
\hline AS & $15.99 \pm 5.10$ & $20.34 \pm 2.86$ & $22.05 \pm 2.74$ & 55.376 & $<.001$ & .283 & MIAEG $<$ IMAEG $<$ IEAEG \\
\hline
\end{tabular}

Nota: MIAEG - Menores Índices de Apoio Emocional Geral; IMAEG - Índices Médios de Apoio Emocional Geral; IEAEG - Índices Elevados de Apoio Emocional Geral; Variáveis Dependentes: BEP (G) - Bem-Estar Psicológico Global; A - Autonomia; DM - Domínio do Meio; CP - Crescimento Pessoal; RP - Relações Positivas; OV - Objetivos de Vida; AS - Aceitação de Si.

Na comparação por sexos, realizada a partir da MANOVA, compreende-se que o sexo masculino apresenta índices superiores face aos Objetivos de Vida, com um valor de efeito fraco $\left[F_{(10,272)}=4.464 ; p=.038\right.$, Wilk's $\lambda=.859$, $\left.\eta_{p}^{2}=.015\right]$. É também significativa a comparação efetuada face à Aceitação de $\mathrm{Si}$, sendo que adolescentes do sexo masculino evidenciam valores mais elevados neste constructo, com um grau de efeito moderado $\left[F_{(10,272)}=\right.$ 4.464; $p=<.001$, Wilk's $\left.\lambda=.859, \eta_{\mathrm{p}}^{2}=.064\right]$. $\mathrm{Na}$ análise às dimensões do PAS, encontram-se evidências que indicam a existência de uma maior perceção de apoio na vertente geral da PAS dos rapazes comparativamente às raparigas, com um efeito fraco $\left[F_{(10,272)}=4.464 ; p=\right.$ .003, Wilk's $\left.\lambda=.859, \eta_{\mathrm{p}}^{2}=.030\right]$.

Com recurso à MANOVA e com a identificação das diferenças entre os grupos a partir do teste Post Hoc (Bonferroni), comparou-se os indivíduos, nos valores médios obtidos para cada dimensão do BEP e PAS, quanto à sua envolvência em ATL, ou não envolvência. Adolescentes envolvidos em práticas associadas ao desporto/ exercício físico apresentam maiores níveis na dimensão Objetivos de Vida, comparativamente aos adolescentes que não praticam atividades de tempos livres, sendo esta razão explicada segundo um grau de efeito fraco $\left[F_{(20,542)}=1.187 ; p=.033\right.$, Wilk's $\left.\lambda=.918, \eta_{\mathrm{p}}^{2}=.024\right]$. Nas dimensões do PAS identifica-se uma maior perceção de suporte geral em adolescentes com envolvência na prática desportiva comparativamente aos estudantes sem prática de tempos livres associada, segundo um efeito fraco $\left[F_{(20,542)}=1.187 ; p=\right.$ .029, Wilk's $\left.\lambda=.918, \eta_{\mathrm{p}}^{2}=.025\right]$.

Na comparação das variáveis dependentes pelos tipos de família, a partir da análise multivariada da variância (MANOVA), depara-se que não existem diferenças significativas para os diferentes tipos de grupo familiar $\left[F_{(30,793)}=\right.$ $.706 ; p>.05$, Wilk's $\lambda=.926]$.

\section{DISCUSSÃO}

O presente estudo teve como propósito comparar níveis de Perceção de Apoio Social (PAS), na sua vertente emocional, de forma a medir o seu impacto preditivo no Bem-Estar Psicológico (BEP) em adolescentes portugueses. Para além da sua compreensão, quisemos contribuir para a discussão em torno dos fatores que podem influenciar o processo de florescimento humano, dada a crescente difusão empírica na área (Fernandes \& Vasconcelos-Raposo, 2008; Fernandes et al., 2011; Reed et al., 2011; Shahimi et al., 2013; Sousa et al., 2012; Tomé et al., 2011). 
Comparou-se ainda o BEP e PAS por sexo, participação em ATL e TF.

Os resultados comparativos indicam que, em regra geral, os grupos com baixa perceção de apoio apresentam níveis significativamente inferiores de BEP, comparativamente aos grupos de perceção média de apoio e aos grupos de perceção alta de apoio social. Tendência semelhante verificou-se na comparação entre o grupo de perceção média de apoio e o de perceção alta de apoio, salvo exceções. Por exemplo, na comparação de grupos por níveis de PAS, a dimensão autonomia, crescimento pessoal e relações positivas apresentaram diferenças significativas entre grupos de menores índices de apoio social face aos grupos médios e elevados, sem diferenças entre os restantes grupos. Idênticas evidências são verificadas nas restantes dimensões de perceção de apoio emocional recebido dos diferentes agentes de interação. Sabendo que a autonomia revela capacidades de autorregulação e autodeterminação do/a adolescente (Fernandes \& Vasconcelos-Raposo, 2008), os nossos resultados apontam que uma baixa perceção de apoio apresenta um impacto negativo direto, neste processo importante para o desenvolvimento. Já o crescimento pessoal apresentou diferenças significativas apenas para os grupos de perceção baixa e alta de apoio dos/as professores/as, com os/as alunos/as do primeiro grupo a apresentarem maiores níveis de crescimento pessoal. $\mathrm{Na}$ comparação por sexo, ao nível do BEP, indivíduos do sexo masculino apresentaram maiores índices nas dimensões objetivos de vida, aceitação de si e PAS. A prática de atividades desportivas e de exercício físico indica melhores índices de objetivos de vida e perceção de suporte geral.

As evidências do estudo sugerem que as perceções mais altas de apoio, que os/as adolescentes percebem receber dos contextos de interação, tenderão a ser benéficas para o seu desenvolvimento adaptativo, ao nível do BEP. De um modo geral, a alta PAS associa-se a níveis mais elevados de BEP, mesmo comparada com alunos/as de grupos intermédios de pereceção, como propõem outros estudos (e.g. Liu \& Yu, 2013), aferindo que o apoio sócio-emocional tende a ser uma mais-valia para o desenvolvimento. Estudos prévios que avaliaram a influência dos grupos sociais e a qualidade de apoio no desenvolvimento de adolescentes, indicam benefícios das relações saudáveis, mantidas entre os/as adolescentes e os contextos diários de interação (Cavalcante et al., 2008; Mota \& Matos, 2010; Rawana, 2013; Shahar et al., 2009; Sidall et al., 2013; Tomé et al., 2011). A crença geral é que o apoio por parte dos/as amigos/as funciona como um elemento protetor contra efeitos nefastos na vida dos/as adolescentes (Pössel et al., 2013; Shahar et al., 2009). Nos vários contextos de investigação, reconhece-se o apoio emocional por parte dos/as amigos/as como um elemento protetor de efeitos nefastos para os/as jovens (Pössel et al., 2013; Shahar et al., 2009). Este estudo adiciona assim informações quanto aos efeitos inerentes da PAS do grupo de amigos ao nível do florescimento humano (Fernandes \& Vasconcelos-Raposo, 2008), numa fase determinante do desenvolvimento, como é a adolescência.

No que concerne ao apoio sócio-emocional da família, este tende a revelar-se determinante para o desenvolvimento positivo do/a jovem (Fernandes et al., 2011; Mombelli et al., 2011; Reed et al., 2011; Siddall et al., 2013), sendo que o mesmo sucede com o impacto do trabalho relacional dos/as professores/as (Pössel et al., 2013). Outras investigações já haviam apurado que a família é um contexto chave para o desenvolvimento positivo do/a adolescente (Fernandes et al., 2011; Mombelli et al., 2011; Reed et al., 2011; Siddall et al., 2013, Tomé et al., 2011). Com os resultados obtidos, trazemos evidências de que há um significativo contributo a dar, por parte deste sistema educativo, no trabalhar de competências como a autonomia ou de formular 
objetivos de vida e outras dimensões do BEP, fundamentais para o desenvolvimento do/a adolescente. Neste sentido, é de todo o interesse o contexto escolar envolver mais a família nos processos formais de ensino-aprendizagem, bem como no trabalho de promoção de competências socioemocionais das crianças e adolescentes, através deste contexto significativo para o desenvolvimento. Quanto aos/ às professores/as, a literatura (Fernandes et al. 2011; Siddall et al., 2013) aponta para associação entre as relações de apoio professor/aaluno/a com a satisfação escolar, sendo que esta apareceu significativamente relacionada ao BEP no estudo de Fernandes et al. (2011). Mota e Matos (2010) também enfatizam a importância do papel do/a professor/a quanto ao desenvolvimento das competências sócio-emocionais do/a adolescente. Pössel et al. (2013), alerta para a influência de aspetos psicossomáticos dos/as adolescentes, na moderação dos efeitos do apoio dos/as professores/as no seu desenvolvimento, sendo que com o presente estudo verificou-se que as competências inerentes ao BEP saem favorecidas se uma maior perceção apoio por parte do/a professor/a para com o/a adolescente estiver patente.

Quanto ao fator sexo, nas comparações pela PAS e BEP e em linha com os nossos resultados, alguns estudos referem que o BEP geral não apresenta diferenças significativas entre sexos, mas sim em dimensões específicas (Fernandes \& Vasconcelos-Raposo, 2008; Freire \& Tavares, 2011; Sousa et al., 2012). Porém, as diferenças apontadas por alguns desses estudos coadunam com os nossos resultados. Por exemplo, Fernandes e Vasconcelos-Raposo (2008) obtiveram resultados semelhantes, associando uma maior aceitação de si aos adolescentes do sexo masculino. Associado à PAS foram identificadas oscilações significativas de efeito fraco, na comparação entre rapazes e raparigas, mas não em dimensões mais específicas, o que pode revelar indícios de transversalidade na aplicação de estratégias promotoras da perceção do apoio social em adolescentes, no trabalho de competências inerentes ao BEP.

A influência da PAS sobre o BEP mostra-se importante considerando que a adolescência constitui um período complexo de relações entre fatores biopsicosocioculturais (Antunes \& Fontaine, 2005; Fernandes \& Vasconcelos-Raposo, 2008). Dada esta evidência apresentamos algumas hipóteses de trabalho e de apoio à intervenção em contextos de apoio ao desenvolvimento humano na adolescência. Sugere-se a implementação de programas de intervenção técnica e especializada, nas escolas e na comunidade, com o objetivo de promover a perceção de apoio e que catalisem relações responsivas (e.g., baseadas em assertividade, empatia, cooperativismo), com vista a criar sinergias entre os agentes educativos (familiares; professores), pares (amigos/as) e o(s) adolescente(s). Acreditamos que será benéfica para todos a utilização de políticas de promoção do BEP, uma vez que este implica fatores como a autonomia, o crescimento pessoal, entre outros, que podem assim diminuir a dependência ou dificuldades de progressão no desenvolvimento socioemocional dos/as adolescentes, com previsíveis impactos ao nível cognitivo e comportamental. Mais que isso, para além de apenas prevenir fenómenos associados à delinquência juvenil (relações adolescente-adolescente) ou à negligência (professores/família- adolescente) cremos que se podem promover competências de relação úteis a todos/as com ganhos para todos/ as. Como refere Tomé et al. (2011), as relações interpessoais mantidas pelos/as adolescentes têm uma grande importância durante o seu desenvolvimento. O equilíbrio entre uma relação positiva com os pais e as mães e com os pares parece ser essencial para um ajustamento positivo dos/as adolescentes (Tomé et al. 2011). Para Mota e Matos (2010) os/as professores/ as constituem-se como figuras importantes no apoio ao desenvolvimento positivo. Deste modo, cremos que uma correta orientação de apoio por parte dos/as professores/as aos/às 
alunos/as constitui um veículo impulsionador dos aspetos cognitivos, não apenas associados à superação dos objetivos curriculares, mas sobretudo numa base educativa de aquisição de competências para a vida, onde as dimensões do BEP exercem um papel importante a ser considerado (Fernandes et al., 2011; Fernandes \& Vasconcelos-Raposo, 2008).

Através dos contributos do estudo, importa refletir sobre algumas limitações. É primeiramente pertinente atender à recolha da amostra, realizada num espetro regional e perante um tipo de amostragem por conveniência e não aleatória, pelo que deve motivar a aprofundamentos empíricos em pesquisas futuras. Pode-se ainda compreender melhor o tipo de avaliação feita com o presente estudo, no que se refere à PAS, motivado pela pouca produção científica associada ao estudo destas variáveis. $\mathrm{O}$ instrumento adaptado por Antunes e Fontaine (1994, 2005) permite avaliar a perceção do/a adolescente do apoio de grupos sociais de interação mensurados no instrumento de um modo generalizado (professores/as, família e amigos/ as). Trabalhos futuros podem procurar identificar agentes específicos que possam amenizar situações em que a perceção de apoio, de dado grupo, constitua fator de risco para o/a adolescente (e.g., pais/mães, encarregados/as de educação, diretor/a de turma). Outra lacuna a explorar relaciona-se com o impacto do suporte não-docente, no meio escolar, ao nível destas caraterísticas. Com o estudo não fica clara a ideia do impacto que os/as funcionários/as dos serviços escolares (e.g., psicólogos/as, administrativos, auxiliares) possam exercer no que se refere à influência da perceção de apoio que os/as adolescentes desenvolvem sobre estes agentes. Conforme já salientado, Mota e Matos (2010) referiram que os/as funcionários/as institucionais podem prestar apoio ativo com lucros psicossociais para os/as adolescentes. Indo ao encontro da literatura (e.g., Siddall et al., 2013), defendemos que os esforços para uma melhoria do contexto escolar não se devem cingir à sala de aula. Será útil investigar o impacto de medidas escolares baseadas nas relações positivas e que envolvam o corpo não docente, procurando medir o impacto do envolvimento e de partilhas sócio-emocionais entre agentes, num contexto mais alargado, criando a hipótese de assim se poder desenvolver o BEP dos/as adolescentes, sem distinção por sexo.

\section{CONCLUSÃO}

Obtivemos resultados significativos que demonstram a influência da PAS ao nível do BEP, em adolescentes portugueses. Especificamente, o grupo de adolescentes com maiores níveis de perceção de apoio apresentam maiores índices nas dimensões do BEP, em geral. Tal evidência confirma a hipótese de que a perceção de apoio emocional tem um impacto positivo importante para o desenvolvimento dos adolescentes ao nível eudamónico que integra o BEP. A perceção de apoio geral, bem como de grupos específicos como o de amigos, professores/as e familiares, sem destinção de tipologia, revela-se essencial para o florescimento humano. Os rapazes apresentam maiores índices de objetivos de vida, aceitação de si e PAS. A envolvência na prática desportiva e de exercício físico revela ter impacto ao nível da formulação de objetivos de vida e na perceção de apoio emocional geral, comparativamente aos adolescentes que não praticam ATL.

É util abordar a importância das figuras e grupos significativos dos adolescentes, onde se podem incluir contextos de interação geral, como o desporto, por forma a promover o desenvolvimento positivo de adolescentes. Embora se trate de uma amostra por conveniência, os nossos resultados vão ao encontro da literatura existente, facto que nos permite indicar a necessidade de se tomarem medidas educativas e de apoio que estimulem relações positivas e promotoras do desenvolvimento do BEP na adolescência. 
Agradecimentos:

Nada declarado.

\section{Conflito de Interesses:}

Nada declarado.

\section{Financiamento:}

Nada declarado.

\section{REFERÊNCIAS}

Antunes, C., \& Fontaine, A. (1994). Diferenças na percepção de apoio social na adolescência: adaptação de uma escala, "social support appraisals". Cadernos de Consulta Psicológica, 10(11), 115-127.

Antunes, C., \& Fontaine, A. (2005). Perceção de apoio social na adolescência: análise fatorial confirmatória da escala Social Support Appraisals. Paidéia, 15(32), 355-366.

Cavalcante, M., Alves, M., \& Barroso, M. (2008). Adolescência, álcool e drogas: uma revisão na perspetiva da promoção da saúde. Escola Anna Nery Revista de Enfermagem, 12 (3), 555-559.

Ciliska, D., \& Wilson, D. (1984). Lifestyle assessment: helping patients change health behaviors. Canadian Family Physician, 30(1), 1665-1670.

Fernandes, H., \& Vasconcelos-Raposo, J. (2008). O bem-estar psicológico em adolescentes: Uma abordagem centrada no florescimento humano. Vila Real: CEDAFESUTAD.

Fernandes, H., Vasconcelos-Raposo, J., Bertelli, R., \& Almeida, L. (2011). Satisfação escola e bem-estar psicológico em adolescentes portugueses. Revista Lusófona de Educação, 18(1), 155-172.

Fernandes, H., Vasconcelos-Raposo, J., Pereira, E., Ramalho, J., \& Oliveira, S. (2009). A influência da atividade física na saúde mental positiva de idosos. Motricidade, 5(1), 33-50. Doi. 10.6063/ motricidade.5(1). 186 .
Fernandes, H., Vasconcelos-Raposo, J., \& Teixeira, C. (2010). Preliminary analysis of the psychometric properties of Ryff's scales of psychological well-being in portuguese adolescents. The Spanish Journal of Psychology, 13(2), 1032-1043. doi: $10.1017 / \mathrm{s} 1138741600002675$.

Freire, T., \& Tavares, D. (2011). Influência da autoestima, da regulação emocional e de género no bem-estar subjetivo e psicológico de adolescentes. Revista de Psiquiatria Clínica, 38(5), 184-188. doi:10.1590/0101-60832011000500003.

Jackson, L., Cumming, S., Drenowatz, C., Standage, M., Sherar, L., \& Malina, R. (2013). Biological maturation and physical activity in adolescent british females: the roles of physical self-concept and perceived parental support. Psychology of Sport and Exercise, 14, 447-454. doi:10.1016.2013.01.001.

Liu, C., \& Yu, C. (2013). Can facebook use induce well-being? Cyberpsychology, Behavior and social Networking, (16)9, 674-678. doi: 10.1089/ 2012.0301.

Mano, M., \& Costa, R. (2011). A influência da inteligência emocional e da prática de exercício físico no bem-estar psicológico dos adolescentes. Tese de Doutoramento, Universidade de Trás-os-Montes e Alto Douro, Vila Real.

Mombelli, M., Costa, J., Marcon, S., \& Moura, C. (2011). Estrutura e suporte familiar como fatores de risco de stresse infantil. Estudos de Psicologia, 28(3), 327-335. doi:10.1590 /S0103166X2011000300004.

Mota, C., \& Matos, P. (2010). Adolescentes institucionalizados: o papel das figuras significativas na predição da assertividade, empatia e autocontrolo. Análise Psicológica, 2 (28), 245-254.

Pössel, P., Rudasill, K., Sawyer, M., Spence, S., \& Bjerg, A. (2013). Associations between teacher emotional support and depressive symptoms in australian adolescents: a 
5-year longitudinal study. Developmental Psychology, 1(1), 1-12. doi: 10.1037/ a0031767.

Rawana, J. (2013). The relative importance of body change strategies, weight perception perceived social support, and self-esteem on adolescente depressive symptoms: longitudinal findinfs from a national sample. Journal of Psychosomatic Reserarch, 75(1), 49-54. doi:10.1016.2013.04.012.

Reblin, M., \& Uchino, B. (2008). Social and emotional support and its implication for health. Current Opinion in Psychiatry, 21 (2), 201-205. doi: 10.1097/YCO.0b013e3282 f3ad89.

Reed, S., Bell, J., \& Edwards, T. (2011). Adolescent well-being in washington state military families. American Journal of Public Health, 101(9), 1676-1682. doi: 10.2105/.2011.300165.

Rees, D., Sabia, J., \& Argys, L. (2009). A head above the rest: height and adolescent psychological well-being. Economics and Human Biology, 7(1), 217-228. doi:10.1016/j.ehb.2009.04.002.

Ryff, C. (1989). Happiness is everything, or is it? Explorations on the meaning of psychological well-being. Journal of Personality and Social Psychology, 57(6), 1069- 1081. doi: 10.1037/00223514.57.6.1069.

Selligman, M., \& Csikszentmihalyi, M. (2000). Positive psychology: na introduction. American Psychologist, 55(1), 5-14. doi: 10.1037/0003-066X.55.1.5.

Shahar, G., Cohen, G., Grogan, K., Barile, J., \& Henrich, C. (2009). Terrorism-Related perceived stress, adolescent depression, and social support from friends. Pediatrics, 124(2), 235-240. doi: 10.1542/peds.20092971.
Shahimi, F., Heaven, P., \& Ciarrochi, J. (2013). The interrelations among the perception of parental styles and psychological wellbeing in adolescence: A longitudinal study. Iranian Journal of Public Health, 42(6), 570-580.

Siddall, J., Heubner, S., \& Jiang, X. (2013). A Prospective study of differential sources of school-related social support and sdolescent global life satisfaction. American Journal of Orthopsychiatry, 83(1), 107-114. doi: 10.1111/ajop.12005.

Sousa, B., Maia, M., \& Vasconcelos-Raposo, J. (2012). Bem-estar, autoestima e índice de massa corporal de adolescentes. Motricidade, 8(2), 1065-1075.

Tomé, G., Camacho, I., Matos, M., \& Diniz, J. (2011). A influência da comunicação com a família e grupo de pares no bem-estar e nos comportamentos de risco nos adolescentes Portugueses. Psicologia: Reflexão e Crítica, 24(4), 747-756. doi:10.15 90/S0102-79722011000400015.

Vasconcelos-Raposo, J. (1993). Os factores psico-sócio-culturais que influenciam $e$ determinam a busca da excelência pelos atletas da elite desportiva portuguesa. Tese de Doutoramento, Universidade de Trás-osMontes e Alto Douro, Vila Real.

Vaux, A. (1988). Social support, theory, research and interventions. New York: Praeger

Zeidner, M., \& Olnick-Shemesh, D. (2010). Emotional intelligence and subjetive well-being revisited. Personality and Indvidual Differences, 48, 431-435. doi: 10.1016/2009.11.011. 